\title{
Spatial Sparsity Based Emitter Localization
}

\author{
Mohammad Pourhomayoun, Student Member, IEEE, Mark L. Fowler, Senior Member, IEEE and \\ N.Eva Wu, Senior Member, IEEE
}

\begin{abstract}
In classical TDOA/FDOA emitter location methods, pairs of sensors share the received data to compute the CAF and extract the ML estimates of TDOA/FDOA. The TDOA/FDOA estimates are then transmitted to a common site where they are used to estimate the emitter location. However, the two-stage method is not necessarily optimal because in the first stage of these methods, the TDOA and FDOA are estimated by ignoring the fact that all measurements should be consistent with a single emitter location. In this paper, we derive a one-stage localization method based on spatial sparsity of the grid plane. In this method, we directly estimate the location of the emitter without going through the intermediate stage of TDOA/FDOA estimation. The Monte Carlo simulation results show that the proposed method has better performance compared to two-stage classic method and also to another available one-stage method named Direct Position Determination (DPD). We will show that the proposed method is also a very effective and beneficial solution to deal with multipath scenarios.
\end{abstract}

Index Terms - Time Difference of Arrival (TDOA), Frequency Difference of Arrival (FDOA), Compressive Sensing (CS), Cross Ambiguity Function (CAF).

\section{INTRODUCTION}

Passive emitter localization is a challenging issue in statistical signal processing. The position can be estimated by measuring one or more location-dependent signal parameters. One of the most popular and common emitter location methods is based on time-difference-of-arrival (TDOA) and frequency-difference-of-arrival (FDOA) estimations. In the classical approach to this method, FDOA and TDOA are estimated from the cross-correlation of the signals received by several pairs of sensors [1]; this is done by computing the cross ambiguity function (CAF) [2] and finding the peak of its magnitude surface. Then these TDOA/FDOA estimates are used in statistical processing to locate the emitter [3].

However the classic two-stage method is not necessarily optimal because in the first stage of these methods, the TDOA and FDOA estimates are obtained by ignoring the fact that all measurements should be consistent with a single emitter

This work was partially supported by the Air Force Research Lab, Rome, NY under contract FA8750-09-2-0068. Approved for Public Release; Distribution Unlimited: 88ABW-2012-0924, 23-Feb-2012 The authors are with Department of Electrical and Computer Engineering, Binghamton Univ., P.O. Box 6000, Binghamton, NY 13902-6000, Phone: 607-777-6973, mpourho1@binghamton.edu,mfowler@binghamton.edu,

evawu@ binghamton.edu. location [4]. In other words, each stage is itself optimal but the cascade of the two stages is not necessarily optimal.

In this paper, we exploit spatial sparsity of the emitter on the $x-y$ plane and use convex optimization theory to estimate the location of the emitter directly without going through the intermediate stage of TDOA/FDOA estimation. It is obvious that in emitter location problems, the number of emitters is much smaller than the number of all grid points in a fine grid on the $x-y$ plane. Thus, by assigning a positive number to each one of the grid points containing an emitter and assigning zeros to the rest of the grid points, we will have a very sparse grid plane matrix that can be reformed as a sparse vector. Since each element of this vector corresponds to one grid point in the $x-y$ plane, we can estimate the location of emitters by extracting the position of non-zero elements of the sparsest vector that satisfies the TDOA/FDOA relationship between transmitted signals and received signals. In principle, sparsity of the grid vector can be enforced by minimizing its $\ell_{0}$-norm (i.e., the number of non-zero elements in the grid vector). However, since the $\ell_{0}$-norm minimization is an NP-hard nonconvex optimization problem, it is very common (e.g in compressive sensing problems) to approximate it with $\ell_{1}$ norm minimization, which is a convex optimization problem and also achieves the sparse solution very well [5]. Thus, after formulating the problem in terms of the sparse grid vector, we can estimate this vector by pushing sparsity using $\ell_{1}$-norm minimization on the grid vector, subject to the TDOA/FDOA relationship between the signals transmitted from the grid point and the signals received by the sensors.

In [6], the authors suggested a source localization method based on TDOA in a multipath channel exploiting the sparsity of the multipath channel for estimation of the line-of-sight component. In this method, the sensors don't need to know the information on the specific transmitted symbols but, they require knowledge of the pulse shape of the transmitted signal. In [7], the authors suggested a compressive-sensing-based distributed target localization using TDOA. In this method, each sensor approximates the transmitted signal by its own received signal mapped to each one of the grid points. This idea helps to reduce the amount of data transmission in the sense of distributed localization but it lowers the quality of the estimation since each sensor estimates the transmitted signal just using its own received signal. Also, each sensor computes its own location estimation that is not necessarily equal to other sensors' estimations. Weiss and Amar [4], [8], [9] developed a single-stage Least-Squares method using TDOA 
and FDOA, named direct position determination (DPD). Kay and Vankayalapati [10] also derived similar results based on a detection theory point of view; the DPD estimator was derived as the ML estimator needed for the generalized likelihood ratio detector. The performance of the DPD method is better than the two-stage classic method (especially for low SNRs). However, the simulation results show that DPD does not obtain accurate results in the case of multipath scenarios.

In this paper, contrary to [6] and [7], we developed a method based on both TDOA and FDOA to take advantage of both delay and Doppler shifts. Contrary to [6], our method does not need any knowledge of the transmitted signal's pulse shape nor any other a priori information. Similar to [7], we exploit the grid point spatial sparsity, but we consider the transmitted signal as a deterministic unknown signal that will be estimated in the sensor network using all received signals. Similar to [10] and [4], we estimate the emitter location directly without going through the intermediate stage of TDOA/FDOA estimation. However, the Monte Carlo simulation results show the higher performance of the proposed method compared to DPD method and classic twostage method especially in multipath scenarios.

\section{PROBLEM FORMULATION}

Suppose that an emitter transmits a signal and $L$ sensors receive that signal. The complex baseband signal observed by the $l^{\text {th }}$ sensor is

$$
r_{l}(t)=\alpha_{l} s\left(t-\tau_{l}\right) e^{j 2 \pi f_{l} t}+w_{l}(t)
$$

where $s(t)$ is the transmitted signal, $\alpha_{l}$ is the complex path attenuation, $f_{l}$ is the Doppler shift, $\tau_{l}$ is the signal delay and $w_{l}(t)$ is a white, zero mean, complex Gaussian noise. Assume that each sensor collects $N_{s}$ signal samples at sampling frequency $F_{s}=1 / T_{s}$. Then, we have

$$
\left.\begin{array}{l}
\boldsymbol{r}_{l}=\alpha_{l} \boldsymbol{W}_{l} \boldsymbol{D}_{l} \boldsymbol{s}+\boldsymbol{w}_{l} \\
\boldsymbol{s} \triangleq\left[\begin{array}{lllll}
s\left(t_{1}\right), & s\left(t_{2}\right), & \ldots & , & s\left(t_{N s}\right)
\end{array}\right]^{T} \\
\boldsymbol{r}_{l} \triangleq\left[\begin{array}{lllll}
r_{l}\left(t_{1}\right), & r_{l}\left(t_{2}\right), & \ldots & , & r_{l}\left(t_{N s}\right)
\end{array}\right]^{T} \\
\boldsymbol{w}_{l} \triangleq\left[\begin{array}{lllll}
w_{l}\left(t_{1}\right), & w_{l}\left(t_{2}\right), & \ldots & , & w_{l}\left(t_{N s}\right)
\end{array}\right]^{T} \\
\boldsymbol{W}_{l} \triangleq \operatorname{diag}\left\{e^{j 2 \pi f_{l} t_{1}}, \quad e^{j 2 \pi f_{l} t_{2}}, \quad \ldots \quad, \quad e^{j 2 \pi f_{l} t_{N s}}\right.
\end{array}\right\}
$$

where $\boldsymbol{r}_{l}$ is the vector containing $N_{s}$ samples of the received signal by $l^{\text {th }}$ sensor, $s$ is $N_{s}$ samples of the transmitted signal, $f_{l}$ is the Doppler shift and $\boldsymbol{D}_{l}$ is the time sample shift operator by $n_{l}=\left(\tau_{l} / T_{s}\right)$ samples. We can write $\boldsymbol{D}_{l}=\boldsymbol{D}^{n_{l}}$ where $\boldsymbol{D}$ is an $N_{s} \times N_{s}$ permutation matrix defined as $[\boldsymbol{D}]_{i j}=1$ if $i=j+1,[\boldsymbol{D}]_{0, N-1}=1$ and $[\boldsymbol{D}]_{i j}=0$ otherwise.

$$
\boldsymbol{D}=\left[\begin{array}{llll}
0 & & & 1 \\
1 & 0 & & \\
& \ddots & \ddots & \\
0 & & 1 & 0
\end{array}\right] \quad, \quad \boldsymbol{D}_{l}=\left[\begin{array}{cccc}
0 & & & 1 \\
1 & 0 & & \\
& \ddots & \ddots & \\
0 & & 1 & 0
\end{array}\right]^{n_{l}}
$$

Now, we assign a number $z_{x, y}$ to each one of the grid points $(x, y)$. Assume that $z_{x, y}$ is one for the grid points containing an emitter and zero for the rest of the grid points. Thus, the signal vector received by $l^{\text {th }}$ sensor will be

$$
\boldsymbol{r}_{l}=\sum_{x} \sum_{y} z_{x, y} \alpha_{l, x, y} \boldsymbol{W}_{l, x, y} \boldsymbol{D}_{l, x, y} \boldsymbol{s}+\boldsymbol{w}_{l},
$$

where $\boldsymbol{W}_{l, x, y}$ and $\boldsymbol{D}_{l, x, y}$ are the Doppler shift and time sample shift operators w.r.t sensor $l$ assuming that the emitter is located in the grid point $(x, y)$ and the summations are over all grid points in the desired $(x, y)$ range. Now, if we reform all of the grid points in a column vector and re-arrange the indices, we will have

$$
\boldsymbol{r}_{l}=\sum_{n=1}^{N} z_{n} \alpha_{l, n} \boldsymbol{W}_{l, n} \boldsymbol{D}_{l, n} \boldsymbol{s}+\boldsymbol{w}_{l}
$$

In (4), we consider the transmitted signal $s$ as a deterministic unknown signal (a common signal model in localization problems). Then, for each grid point, we estimate the transmitted signal using the Minimum Variance Unbiased estimator (MVU) as

$$
\hat{\boldsymbol{s}}_{n}=\frac{1}{L} \sum_{l=1}^{L} \boldsymbol{D}_{l, n}{ }^{-1} \boldsymbol{W}_{l, n}{ }^{-1} \boldsymbol{r}_{l}
$$

where $\hat{\boldsymbol{s}}_{n}$ is the MVU estimate for the transmitted signal from grid point $n$.

We define the matrix $\boldsymbol{\Gamma}_{n}$ as the Doppler and delay operator w.r.t all $L$ sensors, assuming that the received signal comes from the grid point $n$ (there is an emitter at grid point $n)$ :

$$
\boldsymbol{\Gamma}_{n} \triangleq\left[\begin{array}{c}
\alpha_{1, n} \boldsymbol{W}_{1, n} \boldsymbol{D}_{1, n} \\
\alpha_{2, n} \boldsymbol{W}_{2, n} \boldsymbol{D}_{2, n} \\
\vdots \\
\alpha_{L, n} \boldsymbol{W}_{L, n} \boldsymbol{D}_{L, n}
\end{array}\right]_{L N_{s} \times N_{s}}
$$

Then, we can define $\boldsymbol{\theta}_{n}, n \in\{1,2, \ldots, N\}$ as an $L N_{s} \times 1$ vector containing all signals received by all $L$ sensors when the emitter is in grid point $n$ as

$$
\boldsymbol{\theta}_{n} \triangleq \boldsymbol{\Gamma}_{n} \times \hat{\boldsymbol{s}}_{n}
$$


If we arrange all vectors $\boldsymbol{\theta}_{n}$ for $n: 1 \ldots N$ as the columns of a matrix $\boldsymbol{\Theta}$ as

$$
\boldsymbol{\Theta}=\left[\begin{array}{llll}
\boldsymbol{\theta}_{1} & \boldsymbol{\theta}_{2} & \ldots & \boldsymbol{\theta}_{N}
\end{array}\right]_{L N_{s} \times N}
$$

then, we have

$$
\begin{aligned}
& \boldsymbol{r}=\boldsymbol{\Theta} \times \boldsymbol{z}+\boldsymbol{w} \\
& \boldsymbol{r} \triangleq\left[\begin{array}{llll}
\boldsymbol{r}_{1}^{T} & \boldsymbol{r}_{2}^{T} & \ldots & \boldsymbol{r}_{L}^{T}
\end{array}\right]^{T}{ }_{L N_{s} \times 1} \\
& \boldsymbol{z} \triangleq\left[\begin{array}{llll}
z_{1} & z_{2} & \ldots & z_{N}
\end{array}\right]_{N \times 1}^{T}
\end{aligned}
$$

where $\boldsymbol{r}$ is the vector of all $L$ received signals, $\boldsymbol{z}$ is the sparse vector of $z$-values assigned to each grid point and $\boldsymbol{w}$ is the noise. Now, we can solve our problem by forming a BPIC (Basis Pursuit with Inequality Constraints) problem [11] as following:

$$
\left\{\begin{array}{l}
\hat{z}=\arg \min \|z\|_{1} \\
\text { s.t }\|\boldsymbol{\Theta} \times z-\boldsymbol{r}\|_{2} \leq \mathcal{E}
\end{array}\right.
$$

or regularized BPDN (Basis Pursuit Denoising) problem [11] as:

$$
\hat{z}=\arg \min \|\Theta \times z-r\|_{2}+\lambda\|z\|_{1}
$$

\section{SIMULATION RESULTS}

We examined the performance of the proposed method and compared the results using Monte-Carlo computer simulations for different scenarios. In this simulation, the sampling frequency is $80 \mathrm{kHz}$ and the number of samples is equal to 1024 . We assumed that 3 moving sensors receive the signal from one stationary emitter (the location of the emitter has been chosen randomly). Figure (1) shows the RMS Error vs. SNR (with 500 runs for each SNR) for estimating the location of one emitter in $(x-y)$ plane. As we see, the proposed method has better performance compared to DPD and Classic methods.

One of the challenging topics in source localization problems is emitter location estimation in the presence of multipath reflections. We evaluated the capability of the proposed method in dealing with multipath scenarios using Monte-Carlo simulation. Figure (2) shows the RMS Error vs. SNR (with 500 runs for each SNR) for estimating the location of one emitter in multipath case. In this case, we assumed that 4 moving sensors receive the signal from one stationary emitter (the locations of the emitter and reflector points have been chosen randomly). The following plots show better accuracy of the proposed method over DPD and Classic methods. As we see, none of the DPD and Classic methods provide unbiased estimates for higher SNRs.

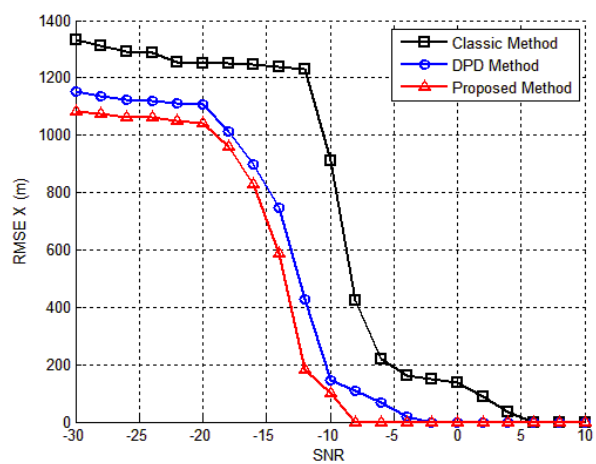

(a)

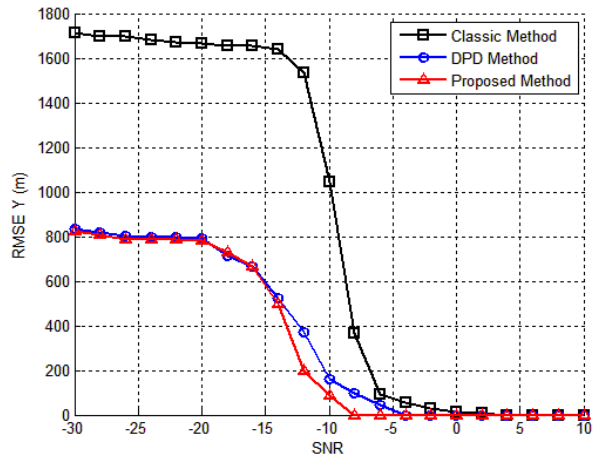

(b)

Figure (1): RMS Error for X and Y (meter) versus SNR (dB) for single-path case.

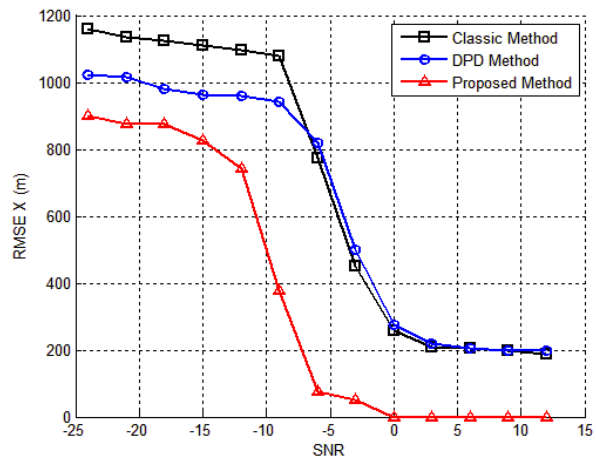

(a)

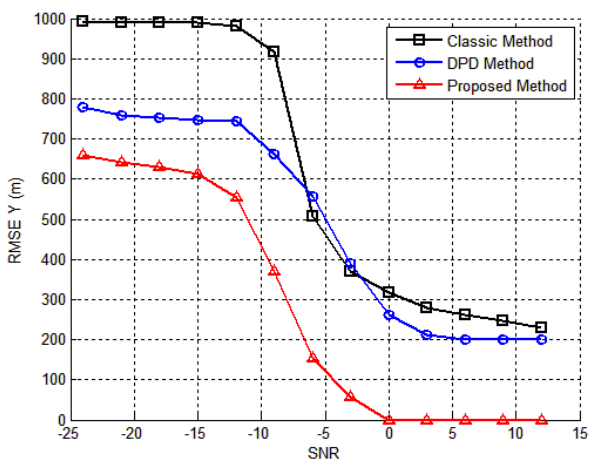

(b)

Figure (2): RMS Error for X and $\mathrm{Y}$ (meter) versus SNR (dB) for multipath scenario. 


\section{CONCLUSION}

We developed a one-stage TDOA/FDOA localization method based on spatial sparsity of emitters. In this method, we assign a non-zero number to each one of the grid points containing an emitter and zero to the rest of the grid points. Thus, the vector formed from these numbers will be a sparse unknown vector that we aim to estimate. Since each element of this vector corresponds to one grid point in $(x, y)$ plane, we can estimate the location of emitters by extracting the position of non-zero elements of the sparsest vector that satisfy the TDOA/FDOA relationship between transmitted signals and received signals. We evaluated the performance of the proposed method using Monte Carlo simulation. Comparing the three curves in each plot in Figures (1) and (2) shows that the proposed method has better performance (especially when there is multi-path) compared to direct position determination (DPD) and two-stage Classic localization methods. Simulation results show that contrary to DPD and Classic methods, the proposed method is a very reliable and strong tool to deal with multipath scenarios.

\section{REFERENCES}

[1] Stein, S., "Differential delay/Doppler ML estimation with unknown signals," IEEE Transactions Signal Process., 41(8), 2717-2719 (1993).

[2] Stein, S. "Algorithms for Ambiguity Function Processing" IEEE Transactions on Acoustics, Speech, Signal Process., 29(3), 588599 (1981).

[3] D. J. Torrieri, "Statistical theory of passive location system," IEEE Transactions on Aerospace and Electronic Systems, vol. AES-20, no. 2, March 1984, pp. 183 - 198.

[4] Weiss, A., Amar, A., "Direct Geolocation of Stationary Wide Band Radio Signal Based on Delays and Doppler Shifts," IEEE Workshop on Statistical Signal Processing, Aug. 31 - Sept. 3, 2009.

[5] R. G. Baraniuk. "Compressive Sensing". IEEE Signal Processing Magazine, 118-120, July 2007.

[6] C.R. Comsa, A.M. Haimovich, S. Schwartz, Y. Dobyns, J.A. Dabin, "Source Localization Using Time Difference Of Arrival Within A Sparse Representation Framework", International Conference on Acoustic, Speech and Signal Process.(ICASP), May 22-27, 2011.

[7] V. Cevher, M. F Duarte, R. G Baraniuk, "Distributed Target Localization Via Spatial Sparsity", $16^{\text {th }}$ European Signal Processing Conference, Switzerland, Aug 2008.

[8] Weiss, A. "Direct Position Determination of Narrowband Radio Frequency Transmitters," IEEE Transactions Signal Process., 11(5), 513-516 (2004).

[9] Amar, A., Weiss, A., "Localization of Narrowband Radio Emitters Based on Doppler Frequency Shifts," IEEE Transactions Signal Process. , 56(11), 5500-5508 (2008).

[10] N. Vankayalapati, S. Kay, "Asymptotically Optimal Detection/Localization of an Emitter of Low Probability of Intercept Signals Using Distributed Sensors," Oct. 2010.

[11] M. F. Duarte, Y. C. Eldar, "Structured Compressed Sensing: From Theory to Applications", IEEE Transactions on Signal Process. , 4053 - 4085, 2011. 